\title{
Strategies for Estimating Genetic Parameters in Marker-Assisted Best Linear Unbiased Predictor Models in Dairy Cattle
}

\author{
S. Neuner, ${ }^{* 1}$ R. Emmerling, ${ }^{*}$ G. Thaller, $†$ and K.-U. Götz ${ }^{*}$ \\ *Bavarian State Research Center for Agriculture, Institute of Animal Breeding, D-85580 Grub, Germany \\ †Christian-Albrechts-University, Institute of Animal Breeding and Husbandry, D-24098 Kiel, Germany
}

\begin{abstract}
An appropriate strategy to estimate variance components and breeding values in genetic models with quantitative trait loci (QTL) was developed for a dairy cattle breeding scheme by utilizing simulated data. Reliable estimates for variance components in QTL models are a prerequisite in fine-mapping experiments and for marker-assisted genetic evaluations. In cattle populations, only a small fraction of the population is genotyped at genetic markers, and only these animals are included in marker-assisted genetic evaluation models. Phenotypic information in these models are precorrected phenotypes [daughter yield deviations (DYD) for bulls, yield deviations (YD) for cows] estimated by standard animal models from the entire population. Because DYD and YD may represent different amounts of information, the problem of weighting these 2 types of information appropriately arises. To detect the best combination of phenotypes and weighting factors, a stochastic simulation for a trait representing milk yield was used. The results show that DYD models are generally optimal for estimating QTL variance components, but properties of estimates depend strongly on weighting factors. An example for the benefit in selection of using YD is shown for the selection among paternal half-sibs inheriting alternative QTL alleles. Even if QTL effects are small, markerassisted best unbiased linear prediction can improve the selection among half-sibs, because the Mendelian sampling variance within family can be exploited, especially in DYD-YD models. Marker-assisted genetic evaluation models should also include YD for cows to ensure that marker-assisted selection improves selection even for moderate QTL effects $(\geq 10 \%)$. A useful strategy for practical implementation is to estimate variance components in DYD models and breeding values in DYD-YD models.
\end{abstract}

Received January 29, 2008.

Accepted June 30, 2008.

${ }^{1}$ Corresponding author: Stefan.Neuner@LfL.bayern.de
Key words: quantitative trait loci, variance component, accuracy of estimated breeding value, markerassisted best linear unbiased prediction

\section{INTRODUCTION}

An adequate approach to combine pedigree and phenotypic information and weighting factors is an essential prerequisite to obtain reliable and unbiased estimates for variance components in QTL models. This is of interest in fine-mapping experiments and for marker-assisted genetic evaluations (MA-BLUP; Fernando and Grossman, 1989) in breeding programs using marker-assisted selection (MAS; Spelman and van Arendonk, 1997).

In contrast to the assumption of nucleus breeding programs in other studies about MAS (Meuwissen and van Arendonk, 1992; Ruane and Colleau, 1996), this study assumed that MAS is added to an existing breeding program. Data from fine-mapping experiments and practical applications of MAS in cattle demonstrate that usually only a small part of the overall population is genotyped. Because only these genotyped animals provide information for QTL-specific evaluations, a 2-step approach has been developed and used to estimate QTL variance components and MA-BLUP EBV (Bennewitz et al., 2004b; Liu et al., 2004; Druet et al., 2006). In the 2-step approach, first, a standard animal model evaluation for the entire population is performed to estimate precorrected phenotypes such as yield deviations (YD) and daughter yield deviations (DYD; Van Raden and Wiggans, 1991). These estimates are then used as observations in an MA-BLUP model for the genotyped animals in the second step. The first implementations of this approach in practical MAS (Liu et al., 2004) have used only information for bulls. Consequently, no information about the EBV of the bull dam contributes to the MA-BLUP EBV of candidates for selection, which are the main focus of MAS breeding programs. This raises the question of how strongly the MA-BLUP EBV for selection candidates are affected, if the dam information is not included in the evaluation model. 
This study examines the combined use of DYD of bulls and YD of cows (bull dams) as observations in MA-BLUP models. Various models were examined to determine the best combination of aggregated phenotypic information (DYD, YD) and weighting factors for the estimation of QTL variance components and MA-BLUP EBV. Weighting factors are applied to account for the different amount of information that is available for the calculation of DYD. Weighting factors commonly used are the variance of DYD (Thaller et al., 2003; Bennewitz et al., 2004a), effective daughter contributions (EDC; Fikse and Banos, 2001), and daughter equivalents (DE; VanRaden and Wiggans, 1991; Druet et al., 2006).

In some situations, the overall accuracies of MABLUP EBV may not be superior to conventional BLUP, because the QTL effects are not large enough. Even in these cases, there will be variance within families attributable to markers, if at least one of the parents is heterozygous at the QTL. As an example for these situations, the benefit of using DYD and YD together in MA-BLUP models for genetic evaluations is shown for the selection among paternal half-sibs inheriting alternative QTL alleles.

\section{MATERIALS AND METHODS}

A stochastic simulation model was applied to evaluate different alternatives for estimating QTL variance components and MA-BLUP EBV in a 2-step approach. Each simulation cycle was divided into 2 phases: data generation and analysis of the simulated data sets.

\section{Data Generation}

In the simulation, a conventional dairy cattle breeding scheme with progeny testing, overlapping generations, and use of proven bulls as sires of second crop daughters was assumed to generate the data. The time horizon for the simulation was $16 \mathrm{yr}$, which is approximately equal to the time span for availability and collection of genotypic data in real research projects. This period consisted of 2 sections. First, a base population was created, and animals were mated randomly to provide a homogeneous population. Subsequently, a breeding program with random selection for the simulated trait was applied to generate data sets. The underlying breeding program is shown in Figure 1. Continuous herd replacement was accounted for, as well as loops and restrictions for the service life of bulls. Parameters of the simulated population and base parameters of the progeny-testing program are shown in Table 1.

In this study, a single trait model for 305-d milk yield was assumed, and we thus simulated a trait with a heritability of 0.36 and an additive genetic variance of $260,100 \mathrm{~kg}^{2}$, according to actual first-lactation parameters of the German Fleckvieh (Interbull, 2007). The overall breeding value (BV) of each animal is the sum of a residual polygenic BV and a QTL BV. With regard to genotypic information, a single biallelic QTL with frequencies of the alternative alleles of 0.5 was assumed. The QTL was linked to a highly informative marker locus consisting of 100 uniformly distributed alleles (polymorphic information content $=0.9899$; proportion of fully informative matings $=0.9799$ ), and the recombination rate between QTL and marker locus was fixed at 0.01. Such a favorable situation at a single marker is not biologically realistic, but it was chosen as a computationally efficient approach to mimic the equivalent information of 4 markers bracketing a postulated QTL position in the middle. Such markers would have 5 alleles each, and the distance between them would be $4 \mathrm{cM}$. The multipoint polymorphic information content (MPIC; Rijsdijk and Sham, 200) of 4 such markers is nearly identical to that of the single marker simulated in our study. All calculations were conducted for different proportions of the QTL variance, accounting for $0,5,10,20,30,40,50$, and $90 \%$ of the overall genetic variance (QTL variance ratio, $\mathbf{Q V R}$ ) in the trait investigated.

\section{Analysis of Simulated Data Sets}

Quantitative trait loci variance components and MABLUP EBV were estimated in a 2-step approach. In the first step, a classical polygenic animal model (AM) evaluation assuming the true variance components to be known was conducted for the entire population to estimate DYD for progeny-tested bulls and YD for cows. Observations in this step were phenotypic records of cows. In typical real-life situations, all animals are included in genetic evaluations of dairy cattle, but only a small fraction of animals might be genotyped at genetic markers. These animals are most likely proven bulls, bull dams, and selection candidates for progeny testing. Because only genotyped animals can provide information for the estimation of QTL variance components and MA-BLUP EBV, the second evaluation step was only applied to this genotyped subset of the population. For the current study, we assumed that marker information was available for all animals in the MA-BLUP pedigree and known without error. Observations used in step 2 were twice the DYD and YD calculated in step 1. Twice the DYD was used as phenotypic information of bulls in all MA-BLUP models in the current study, because twice the DYD contain the complete additive genetic variance, and estimates of different models can be compared directly. 
Table 1. Characteristics of the cow population, bull population, and the breeding program

\begin{tabular}{lr}
\hline Cow population & \\
\hline Milking cows, no. & 20,000 \\
Cows in lactation, \% & 35 \\
Lactation 1 & 27 \\
Lactation 2 & 21 \\
Lactation 3 & 17 \\
Lactation 4 & 250 \\
Bull dams, no. & 24 \\
Age at first calving, mo & 12 \\
Intergestation period, mo & \\
AI bull population & 24 \\
Age at birth of first progeny, mo & 60 \\
Age at first breeding value estimation, mo & 48 \\
Service life as proven bull, mo & 108 \\
Maximum age, mo & 72 \\
Bulls sampled, no./yr & 10 \\
Sires for insemination service per year, no. & 4 \\
Sires of bulls used per year, no. & 70 \\
Daughter records per bull sampled, no. & \\
\hline
\end{tabular}

The pedigree used for MA-BLUP evaluations contains all progeny-tested bulls, waiting bulls, young bulls currently used, and young bull candidates for the subsequent years. All these animals are included with 3 generations of ancestors. The number of animals for MA-BLUP calculations was therefore about 5,500 individuals, consisting of 1,200 proven bulls with DYD, 3,800 cows with $\mathrm{YD}$, and 500 young bulls.

The MA-BLUP model of Fernando and Grossman (1989) was used for evaluations:

$$
y_{i}=\mu+u_{i}+v_{i}^{p}+v_{i}^{m}+e_{i},
$$

where $y_{i}=$ the record (YD for dams and twice the DYD for sires) of individual $i$; $u_{i}=$ the residual polygenic effect of individual $i ; v_{i}^{p}$ and $v_{i}^{m}=$ the paternal and maternal gametic effects of individual $i$; and $e_{i}=$ the residual. Gametic effects were included in the evaluations in terms of the identical-by-descent matrix. The identical-by-descent matrices were calculated following the algorithm of Abdel-Azim and Freeman (2001).

For estimating AM EBV, DYD, and YD, the package MIX99 (Vuori et al., 2006) was used. Parameters for MA-BLUP models and MA-BLUP EBV were estimated with the ASREML package (Gilmour et al., 1995).

In addition to the AM and MA-BLUP models, EBV were calculated using a classical AM for the decreased data set. This model had only 1 predictor for the overall animal effect, and it is henceforth denoted as AM on MA-BLUP records (AM-MA). For the AM-MA and the MA-BLUP models, various combinations of phenotypic information (DYD, YD) and weighting factors were considered. The evaluations were divided into blocks
$\mathrm{A}$ and $\mathrm{B}$, which are characterized by the phenotypic information used. Block $\mathrm{A}$ is similar to the German Holstein MA-BLUP system (Liu et al., 2004), in which only DYD (DYD models) are used, whereas in block B, DYD and YD (DYD-YD model) are used together, similar to the French MA-BLUP system (Boichard et al., 2002). Within the blocks, different weighting factors, as described in the literature, were applied to DYD: no weighting, variance of DYD (Bennewitz et al., 2004a), EDC (Fikse and Banos, 2001; Liu et al., 2004; Szyda et al., 2005), and DE (VanRaden and Wiggans, 1991; Druet et al., 2006). Yield deviations were not weighted, because each cow had only 1 record in the current study (T. Druet, Station de Génétique Quantitative et Appliquée, Institut National de la Recherche Agronomique, Jouy-en-Josas, France, personal communication). Table 2 summarizes the different variants.

When using DYD and YD together in the same model, one must note that these types of information contain different amounts of genetic and residual variance, even if twice the DYD are used. Hence, their scales are not identical. A detailed description of this issue is shown in the appendix. An additional weighting factor $\gamma$ was derived for DYD to combine DYD and YD in a single evaluation model by accounting for the different amounts of genetic and residual variances that DYD and YD contain. Results for using the additional weight $\gamma$ are shown for a QVR of 0.30 .

The analysis of benefits of MA-BLUP for the selection among paternal half-sibs was done for a simulated data set with a QVR of 0.10 . Progeny of heterozygous sires were divided into 2 groups: those inheriting the favorable allele 1 and those inheriting the unfavorable allele 0. Then their simulated BV, their AM EBV, and MA-BLUP EBV were compared.

\section{RESULTS}

\section{Variance Component Estimation}

The properties of the variance component estimations were evaluated by comparing the absolute values for the estimated components and the estimated QVR with the simulated parameters. The results obtained were consistent for all simulated QVR. For demonstration, the results of the variance component estimation for a simulated QTL ratio of 30\% are shown in Table 3. The figures represent averages of 25 replicates. Results clearly show that the use of DYD models is superior to that of DYD-YD models. The absolute deviation of the estimated genetic variances and QVR from the simulated parameters is smallest when using DE or EDC as weighting factors in DYD models. In DYD-YD MA-BLUP models, the estimated variance components 
Table 2. Evaluations applied to the simulated data sets ${ }^{1}$

\begin{tabular}{llll}
\hline & & \multicolumn{2}{c}{ Evaluation model } \\
\cline { 2 - 4 } $\begin{array}{l}\text { Properties, data } \\
\text { considered }\end{array}$ & AM & AM-MA & MA-BLUP \\
\hline Animals included & Entire population & MA-BLUP data set & MA-BLUP data set \\
Phenotypic information & Milk yield records & A: DYD & A: DYD \\
& of adult females & B: DYD + YD & B: DYD + YD \\
& & Weighting factors applied to & Deighting factors applied to \\
& & DYD in block A and B: & -no weighting \\
& & -no weighting & -variance of DYD \\
& & -variance of DYD & -EDC \\
\hline
\end{tabular}

${ }^{1}$ Applied evaluation models are the animal model (AM), the animal model on marker-assisted-BLUP data (AM-MA), and the marker-assisted BLUP model (MA-BLUP). For AM-MA and MA-BLUP models, only daughter yield deviations (block A, DYD) or daughter yield deviations in combination with yield deviations (block B, DYD-YD) were used as phenotypic information. Calculations were done without weighting of DYD, weighting DYD by the variance of DYD, effective daughter contributions (EDC), and daughter equivalents (DE).

were more biased; however, the weighting factor based upon variance of DYD seemed to be optimal in this case. Thus, weighting of information was essential for block $\mathrm{A}$ and $\mathrm{B}$, because unweighted daughter information caused a severe overestimation of variance components in all cases. The very large phenotypic variances for DYD models in Table 3 are caused by using twice the DYD and applying different weighting factors. The expected variance of twice the DYD is derived in the appendix. If DE or EDC are used as weighting factors, the expected phenotypic variance equals $2,629,900$ $\mathrm{kg}^{2}$.

Table 4 shows the results for a QVR of 0.10 and the EDC weighting factor multiplied by an additional weighting factor $\gamma$. In the DYD model (block A), only the phenotypic variance changed toward the simulation parameter if $\gamma$ was applied. Using $\gamma$, it was possible to estimate the ordinary phenotypic variance as expected. Including $\gamma$ in the weighting factor for the DYD-YD model, the additive genetic variance was no

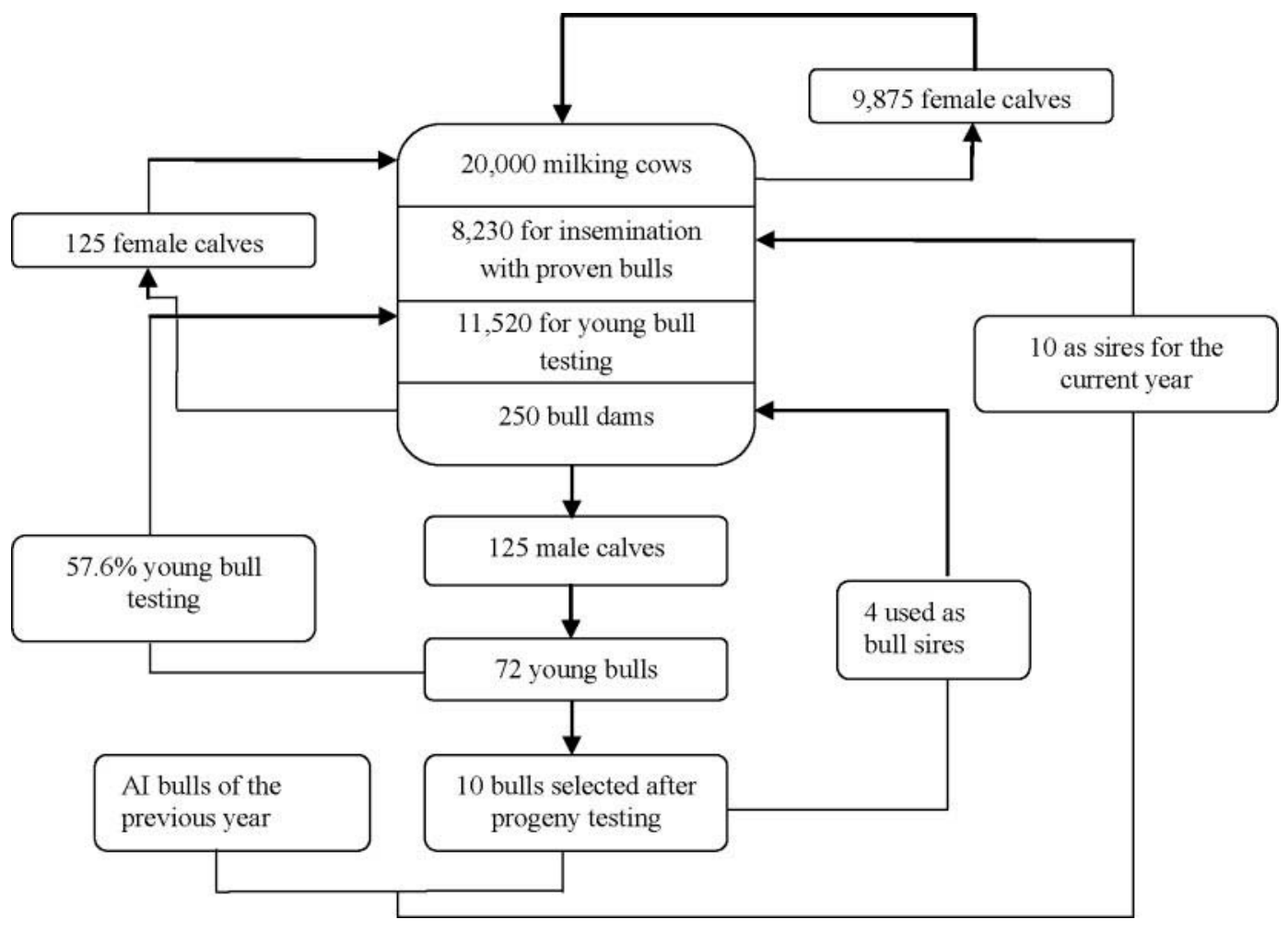

Figure 1. Population structure and breeding strategy, indicating the flow of genes and multistage selection. 
Table 3. Simulated and estimated values of variance components for a QTL variance of 30\% compared with the total genetic variance (QTL variance ratio, $\mathrm{QVR})^{1}$

\begin{tabular}{|c|c|c|c|c|c|c|}
\hline Block & $\begin{array}{l}\text { Weighting } \\
\text { factor }\end{array}$ & $\begin{array}{l}\text { Additive genetic } \\
\text { variance }\end{array}$ & $\begin{array}{l}\text { Phenotypic } \\
\text { variance }\end{array}$ & $\begin{array}{l}\text { Residual polygenic } \\
\text { variance }\end{array}$ & QTL variance & QVR \\
\hline \multirow[t]{3}{*}{ A (DYD) } & No & 310,276 & 302,749 & 226,130 & 84,146 & 0.271 \\
\hline & $\mathrm{DE}$ & 267,523 & $2,636,408$ & 189,122 & 78,401 & 0.293 \\
\hline & EDC & 266,468 & $2,372,263$ & 187,311 & 79,157 & 0.297 \\
\hline \multirow[t]{2}{*}{ B (DYD-YD) } & No & 332,366 & 633,458 & 240,387 & 91,979 & 0.277 \\
\hline & $\mathrm{DE}$ & 296,924 & 735,948 & 221,844 & 75,080 & 0.253 \\
\hline \multicolumn{2}{|c|}{ Simulated parameters } & 260,100 & 722,500 & 182,070 & 78,030 & 0.300 \\
\hline
\end{tabular}

${ }^{1}$ Calculations were done by marker-assisted BLUP models that use only daughter yield deviations (block A, DYD) or daughter yield deviations in combination with yield deviations (block B, DYD-YD) as phenotypic information. Daughter yield deviations were not weighted (No), weighted by the variance of DYD [var(DYD)], effective daughter contributions (EDC), and daughter equivalents (DE). Values shown are the averages over 25 replicates.

longer overestimated, and the estimates for the genetic variances were similar to the findings for DYD models.

\section{Accuracy of MA-BLUP EBV}

For each group of animals (bulls, cows, and young bulls), correlations between true BV and EBV were calculated for all models. Similar to the estimation of variance components, the findings were consistent for all simulated QVR. Correlations between EBV and simulated BV for a QVR of 30\% are shown in Table 5. The results are the averages of 25 replicates.

Our results show that weighting with DE or EDC is also beneficial for the estimation of MA-BLUP EBV. Furthermore, the differences of the correlations are small, no matter which weighting factor is applied. Correlations of proven bulls are nearly unaffected by the inclusion of YD in the evaluation models. In contrast to proven bulls, correlations for bull dams and young bulls in the AM-MA and the MA-BLUP model highly depend on whether YD are included in the phenotypic information (block B) or not (block A). If YD are not included, no observations for bull dams are considered and their EBV are based only on pedigree information in the MA-BLUP data. Because EBV of young bulls are calculated based on only the EBV of their parents, the advantage of using YD is obvious. For a QVR of 30\%, the difference in correlations between DYD-YD models and DYD models is about 0.054 for young bulls and 0.226 for the cows. Furthermore, if only DYD are used in MA-BLUP evaluations (block A), a QTL representing at least $30 \%$ of the additive genetic variance is needed to get accuracies for young bulls in MA-BLUP models that exceed the accuracy of the pedigree BV from the classical AM. In DYD-YD MA-BLUP models (block B), QVR larger than $10 \%$ are sufficient to obtain greater accuracies for young bulls compared with conventional genetic evaluations. In Figure 2, we present the results for all calculated models and QVR.

Lower correlations for all animal groups in AM-MA compared with AM for all scenarios (Table 5 and Figure 2) indicate that there is a loss of information if 2 -step approaches are applied. Analogous to the estimation

Table 4. Simulated and estimated values of variance components for a QTL variance of 30\% compared with the total genetic variance (QTL variance ratio, QVR) if the additional weight $\gamma$ is applied to daughter yield deviations ${ }^{1}$

\begin{tabular}{|c|c|c|c|c|c|c|}
\hline Block & $\begin{array}{l}\text { Weighting } \\
\text { factor }\end{array}$ & $\begin{array}{l}\text { Additive genetic } \\
\text { variance }\end{array}$ & $\begin{array}{l}\text { Phenotypic } \\
\text { variance }\end{array}$ & $\begin{array}{l}\text { Residual polygenic } \\
\text { variance }\end{array}$ & QTL variance & QVR \\
\hline \multirow[t]{2}{*}{$\mathrm{A}(\mathrm{DYD})$} & $\mathrm{EDC}$ & 257,857 & $2,664,154$ & 175,317 & 82,540 & 0.320 \\
\hline & $\mathrm{EDC} \times \gamma$ & 257,881 & 727,459 & 175,337 & 82,545 & 0.320 \\
\hline B (DYD-YD) & $\mathrm{EDC} \times \gamma$ & 259,730 & 713,409 & 178,590 & 81,140 & 0.312 \\
\hline \multicolumn{2}{|c|}{ Simulated parameters } & 260,100 & 722,500 & 182,700 & 78,030 & 0.300 \\
\hline
\end{tabular}

${ }^{1}$ Calculations were done by marker-assisted BLUP models that use only daughter yield deviations (block A, DYD) or daughter yield deviations in combination with yield deviations (block B, DYD-YD) as phenotypic information. Weighting factors applied to DYD were effective daughter contributions (EDC) and effective daughter contributions multiplied by $\gamma($ EDC $\times \gamma)$. Reported values are the averages over 25 replicates, 
Table 5. Correlations between simulated and estimated breeding values for progeny-tested bulls, bull dams (cows), and selection candidates for progeny testing (young bulls) for a QTL variance of $30 \%$ compared with the total genetic variance ${ }^{1}$

\begin{tabular}{|c|c|c|c|c|c|c|c|c|c|c|}
\hline \multicolumn{2}{|c|}{$30 \% \mathrm{QTL}$} & \multicolumn{3}{|c|}{ Progeny-tested bulls } & \multicolumn{3}{|c|}{ Cows } & \multicolumn{3}{|c|}{ Young bulls } \\
\hline Block & $\begin{array}{l}\text { Weighting } \\
\text { factor }\end{array}$ & $\mathrm{AM}$ & AM-MA & MA-BLUP & $\mathrm{AM}$ & AM-MA & MA-BLUP & $\mathrm{AM}$ & AM-MA & MA-BLUP \\
\hline \multirow{3}{*}{ A (DYD) } & $\mathrm{DE}$ & & 0.908 & 0.913 & & 0.466 & 0.489 & & 0.531 & 0.602 \\
\hline & $\operatorname{var}(\mathrm{DYD})$ & & 0.907 & 0.912 & & 0.465 & 0.489 & & 0.531 & 0.602 \\
\hline & EDC & & 0.908 & 0.913 & & 0.466 & 0.489 & & 0.531 & 0.602 \\
\hline \multirow{2}{*}{ B (DYD-YD) } & $\operatorname{var(DYD)}$ & & 0.864 & 0.864 & & 0.692 & 0.702 & & 0.561 & 0.623 \\
\hline & $\mathrm{EDC}$ & & 0.912 & 0.917 & & 0.704 & 0.715 & & 0.586 & 0.656 \\
\hline
\end{tabular}

${ }^{1}$ Calculations were done by marker-assisted BLUP (MA-BLUP) models that only use daughter yield deviations (block A, DYD) or DYD in combination with yield deviations (block B, DYD-YD) as phenotypic information. Daughter yield deviations were not weighted (No), weighted by the variance of DYD [var(DYD)], effective daughter contributions (EDC), and daughter equivalents (DE). Applied evaluation models are the animal model (AM), the animal model on MA-BLUP data (AM-MA), and the MA-BLUP model (MA-BLUP). Depicted accuracies are the averages over 25 replicates.

of variance components, we applied the additional weight $\gamma$ to DYD for the estimation of MA-BLUP EBV. The results for a QVR of 0.30 are presented in Table 6 . In block A, no changes in accuracies were observed whether $\gamma$ was applied or not, and accuracies increased only slightly in block B.

Besides the accuracies, also the variance of the EBV for each group of animals was investigated. This analysis was done to check whether the estimates from the original AM and the MA-BLUP evaluations can be compared with each other without additional data transformation or standardization. Our findings are shown in Table 7. Similar to Table 6, no differences in the results for block A were observed. In block B, the variance of EBV increased remarkably from AM to AM-MA and MA-BLUP models, if $\gamma$ was not applied. Otherwise, the variances of the EBV were in better agreement with the expectations for observed accuracies in Tables 5 and 6 and the simulated additive genetic variance.

\section{Half-Sib Selection}

To illustrate the benefit of using YD and to investigate whether MA-BLUP is beneficial even for low to moderate QTL effects, progeny of heterozygous sires (half-sibs) were analyzed from a scenario with 10\% QVR. Accuracies of all young bulls for a QVR of $10 \%$ were 0.599 in the AM, 0.551 in the MA-BLUP DYD model, and 0.606 in the MA-BLUP DYD-YD model. In conclusion, the overall accuracies of MA-BLUP EBV were only slightly superior to conventional BLUP in DYD-YD models, and there was a substantial increase from MA-BLUP DYD to DYD-YD models.

If only progeny of heterozygous sires were considered, it was found that progeny inheriting the favorable QTL allele had on average $216 \mathrm{~kg}$ greater true BV. In MA-BLUP models their EBV were 30 and $46 \mathrm{~kg}$ higher without and with the use of YD, respectively. In other words, the use of YD allowed for increased distinction of the progeny with different QTL alleles inherited

Table 6. Correlations between simulated and estimated breeding values for progeny-tested bulls, bull dams (cows), and selection candidates for progeny testing (young bulls) for a QTL variance of $30 \%$ compared with the total genetic variance if the additional weight $\gamma$ is applied to daughter yield deviations ${ }^{1}$

\begin{tabular}{|c|c|c|c|c|c|c|c|c|c|c|}
\hline \multicolumn{2}{|c|}{$30 \% \mathrm{QTL}$} & \multicolumn{3}{|c|}{ Progeny-tested bulls } & \multicolumn{3}{|c|}{ Cows } & \multicolumn{3}{|c|}{ Young bulls } \\
\hline Block & $\begin{array}{l}\text { Weighting } \\
\text { factor }\end{array}$ & $\mathrm{AM}$ & AM-MA & MA-BLUP & $\mathrm{AM}$ & AM-MA & MA-BLUP & $\mathrm{AM}$ & AM-MA & MA-BLUP \\
\hline B (DYD-YD) & $\begin{array}{l}\text { EDC } \times \gamma \\
\text { EDC }\end{array}$ & 0.917 & $\begin{array}{l}0.906 \\
0.908\end{array}$ & $\begin{array}{l}0.910 \\
0.912\end{array}$ & 0.740 & $\begin{array}{l}0.461 \\
0.702\end{array}$ & $\begin{array}{l}0.484 \\
0.712\end{array}$ & 0.582 & $\begin{array}{l}0.523 \\
0.576\end{array}$ & $\begin{array}{l}0.573 \\
0.627\end{array}$ \\
\hline & $\mathrm{EDC} \times \gamma$ & & 0.910 & 0.915 & & 0.702 & 0.713 & & 0.577 & 0.628 \\
\hline
\end{tabular}

${ }^{1}$ Calculations were done by marker-assisted BLUP (MA-BLUP) models that only use daughter yield deviations (block A, DYD) or DYD in combination with yield deviations (block B, DYD-YD) phenotypic information. Weighting factors applied to DYD were effective daughter contributions $(\mathrm{EDC})$ and effective daughter contributions multiplied by $\gamma(\mathrm{EDC} \times \gamma)$. Applied evaluation models are the animal model $(\mathrm{AM})$, the animal model on MA-BLUP data (AM-MA), and the MA-BLUP model (MA-BLUP). Depicted accuracies are the averages over 25 replicates. 

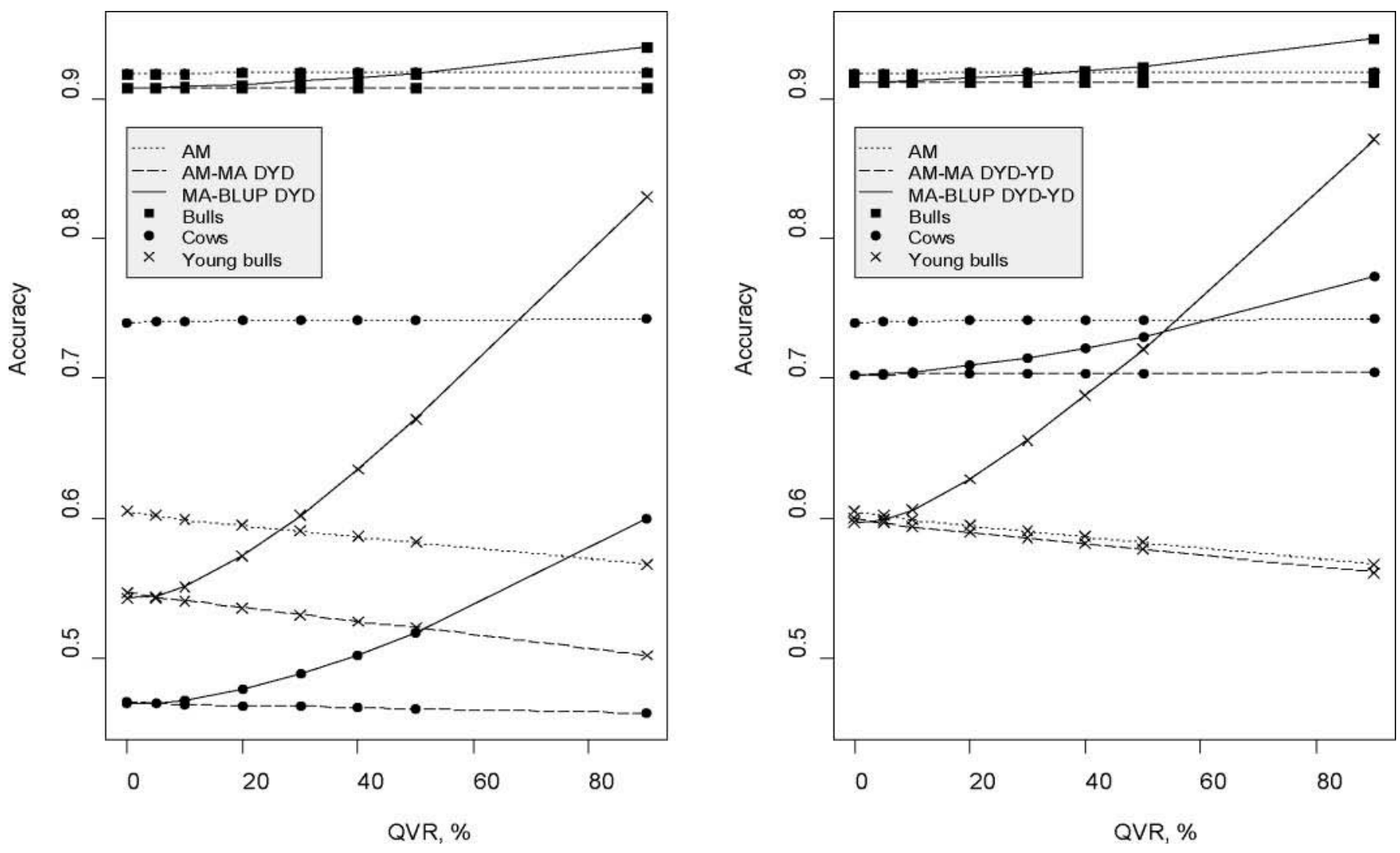

Figure 2. Accuracies of estimated breeding values for a QTL variance of 0, 5, 10, 20, 30, 40, 50, and 90\% compared with the total genetic variance (QTL-variance ratio, QVR) in conventional animal model evaluations (AM), animal model evaluations on marker-assisted-BLUP data (AM-MA) and marker-assisted-BLUP evaluation models (MA-BLUP). On the left, results are depicted for MA-BLUP data sets that use only daughter yield deviations (DYD) of bulls as phenotypic information, whereas on the right, DYD of bulls and yield deviations (YD) of cows are used together in MA-BLUP data sets. Weighting factors applied to DYD were effective daughter contributions. The curves show the accuracies for progeny-tested bulls $(\mathbf{\bullet})$, cows $(\bullet)$, and young bulls $(\times)$ for the AM (dotted line), the AM-MA (dashed line), and the MA-BLUP (solid line) evaluations.

by their sire. As expected, no differences were found among the progeny of nonsegregating sires.

\section{DISCUSSION}

One objective of MAS in dairy cattle breeding programs is the optimized selection of young bulls for performance testing. This goal can only be achieved if the accuracy of MA-BLUP EBV is greater than the accuracy from conventional BLUP.

A precondition for MAS is the ability to trace the transmission of QTL alleles through the pedigree. In practical research, multiple markers surrounding a QTL make it possible to infer the transmission of QTL alleles with great precision if the data are complete and polymorphic markers are chosen. In our study, only a single highly polymorphic marker was used to mimic a multiple-marker situation to decrease computational costs for data simulation and MA-BLUP evaluations.
Such a marker is unlikely to exist in real life, however. To affirm the assumption that a single marker can be used to mimic multiple markers, the MPIC (Rijsdijk and Sham, 2002) for a situation of 4 equally spaced markers that bracket a QTL was calculated. Each marker had 5 alleles, and the distance between the markers was $4 \mathrm{cM}$. The MPIC for a QTL position centered between the 2 markers in the middle of the marker bracket was 0.912. Using our parameters of the single marker, the corresponding MPIC at the QTL position was 0.914 .

In most cases, practical applications of MA-BLUP are based on a 2-step approach as described by Liu et al. (2004) and Druet et al. (2006). Up to now, EDC (Liu et al., 2004) and DE (Druet et al., 2006) have been used as weighting factors for MA-BLUP evaluations. One focus of the current study was to appropriately weight the daughter information in DYD and DYD-YD models. Our results show that only correct weights accounting for the varying amount of information available for the 
phenotypes YD and DYD ensure unbiased estimates of variance components and, therefore, high accuracies of MA-BLUP EBV. With this respect, it seems that the correct choice of the weighting factor is more important when estimating variance components, to obtain the correct estimates, than for the estimation of MABLUP EBV. By setting up the mixed model equations for BLUP, one can see that the correct ratios of the genetic variance components to each other and to the residual variance component are more important than to their absolute values, especially if accuracies are considered. Greatest accuracies are always obtained if the estimated variance components and ratios are closest to the simulated parameters.

Weighting is especially difficult for variance component estimation in DYD-YD models, because the scales of the 2 types of information are not identical. We showed that the estimates obtained from DYD-YD models can be substantially improved by using the additional weight $\gamma$. Otherwise, a considerable overestimation of the total additive genetic variance occurs. As a consequence, we strongly recommend to correct for different amounts of genetic and residual variances when combining DYD and YD for variance component estimation. If variance component estimates from DYD-YD MA-BLUP models without $\gamma$ are further used for MA-BLUP DYD-YD evaluations, the variances of the EBV will be overestimated.

To validate this observation, the expected variance of the EBV can be calculated by multiplying the simulated additive genetic variance by the squared observed accuracies. Deviations between the expected variances and the observed values with correction were between $0 \%$ for bulls and $1 \%$ for young bulls. If the correction was not applied, the variance of EBV for young bulls was too large. For illustration, the expected variance of EBV for young bulls and a QVR of 0.30 was 102,253 $\mathrm{kg}^{2}\left(0.627^{2} \times 260,100 \mathrm{~kg}^{2}\right)$, but the observed value was
$116,434 \mathrm{~kg}^{2}$ (Table 7). This result corresponds to an overestimation of $14 \%$.

Another advantage for EBV calculated in DYD-YD models using $\gamma$ is that they can directly be compared with EBV from an AM without additional postprocessing (standardization) of the EBV. This is important in practical applications, because the subset of results from MA-BLUP EBV must be comparable to all other EBV from the AM.

Applying 2-step approaches for MA-BLUP models always causes a loss of information. Because only a small fraction of the population is included in the analysis, several relationships among animals are not accounted for in MA-BLUP data sets. The information content for proven bulls decreases only marginally, because DYD are estimated very accurately from many daughters and parents contribute only marginally to their EBV. For cows, only (male) relationships and, in the case of DYD-YD models, their own corrected phenotypes are taken as sources of information. As a consequence, the accuracies of cows were decreased from 0.742 to 0.466 in AM-MA without YD, and the use of MA-BLUP could only compensate a very small part of this. If YD were included in the model, the accuracy for cows again increased up to 0.704 . In consequence, the loss of information due to the 2-step approach and to missing phenotypes of bull dams in DYD models has to be overcome by an additional source of information: QTL information. Our results show that with an appropriate model, the compensation of the loss of accuracy requires a QTL explaining at least $10 \%$ of the genetic variance of the trait. Analyses of MAS applied to practical breeding programs describe the increase in accuracies of young bulls (Liu et al., 2004; Druet et al., 2005). Liu et al. (2004) described the increase in accuracy of young German Holstein bulls if 2 QTL were included as random effects and DGAT1 (Grisart et al., 2002; Winter et al., 2002) as a fixed effect in MA-BLUP evaluation. Cor-

Table 7. Variances of estimated breeding values for progeny-tested bulls, bull dams (cows), and selection candidates for progeny testing (young bulls) for a QTL variance of $30 \%$ compared with the total genetic variance if the additional weight $\gamma$ is applied to daughter yield deviations $^{1}$

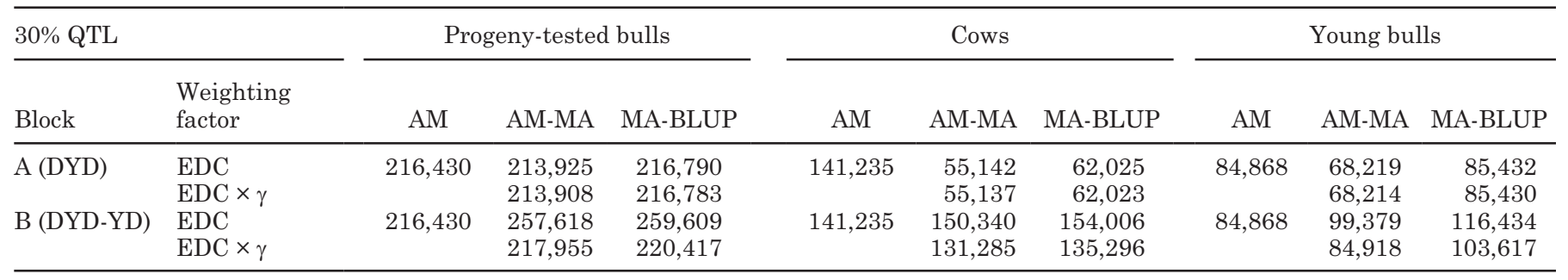

${ }^{1}$ Calculations were done by marker-assisted BLUP (MA-BLUP) models that only use DYD (block A, DYD) or DYD in combination with yield deviations (block B, DYD-YD) as phenotypic information. Weighting factors applied to DYD were effective daughter contributions (EDC) and effective daughter contributions multiplied by $\gamma(\mathrm{EDC} \times \gamma)$. Applied evaluation models are the animal model (AM), the animal model on MABLUP data (AM-MA), and the MA-BLUP model (MA-BLUP). The values shown are the averages over 25 replicates. 
relations increased from 0.45 in the AM-MA to 0.65 in the MA-BLUP model, mainly due to DGAT1. However, more important than comparing results of AM-MA and MA-BLUP models is the superiority of accuracies from MA-BLUP models over traditional AM. Druet et al. (2005) investigated this for the French MAS program. In the French MAS system, 40 to $50 \%$ of the variance for all milk traits is explained by 3 to 5 QTL. Accuracies for milk yield EBV of young bulls increased from 0.47 to 0.55 . Results of our analysis for a 40\% QTL showed an increase from 0.58 to 0.68 . These differences can be explained by different heritabilities and different designs for the French breeding program and the one assumed in our study. In addition, the very favorable properties of the QTL and the markers in our study lead to a higher level of accuracies.

Further investigations are necessary regarding the phenotypic information used in DYD-YD MA-BLUP models. Daughter YD were not corrected for bull dams having a YD in DYD-YD models to avoid double counting. Because our analyses were based on random selection, and because each bull had at least 70 daughters with records, the effect of double counting was assumed to be small. Even more gain is expected if the YD of a bull dam and information of its parents and progeny is combined as phenotypic information for bull dams. Another issue that has to be noted in the context of phenotypic information used in DYD-YD MA-BLUP is the effect of preferential treatment of bull dams on MA-BLUP EBV. If there is any preferential treatment of bull dams, this problem arises in both conventional AM evaluations and MA-BLUP DYD-YD models. One approach to decrease this problem is the consideration of heterogeneous variances in the AM evaluations, which should lead to less biased YD for bull dams in MA-BLUP. In case of a preferential treatment, the superiority of DYD-YD models over DYD models will decrease. According to our results, it seems to be preferable to accept a small possible bias rather than discard the phenotypic information of bull dams completely.

The general choice of whether DYD or DYD-YD models are to be preferred depends on the intention of the research. In fine-mapping of QTL, one is especially interested in correct estimates of variance components, whereas MAS aims at an increase in accuracies for MABLUP BV of animals without their own phenotypic or progeny information. Therefore, DYD models should be chosen for the estimation of variance components if it is not possible to derive the correct weighting factors to combine DYD and YD correctly. One way to derive the weighting factors for the assumptions made in this study is shown in the appendix. The importance of correct parameter estimates explained by a QTL for MAS was shown by Ruane and Colleau (1996) and Spelman and van Arendonk (1997). They calculated the genetic gain with correct parameter estimates for the selection for a nonexistent QTL and for the selection with an overestimated QTL variance. Both situations of incorrect parameter estimates resulted in less genetic gain compared with the results for correct parameter estimates. To achieve an increase in accuracies for MA-EBV of animals without their own phenotypic or progeny information, MA-BLUP models for MAS should include both DYD and YD to ensure the greatest possible efficiency of selection.

In addition to our empirical results, the benefit of using YD of dams can be shown by a simple calculation. The accuracy of the EBV of a young bull candidate $\left(r_{Y B}\right)$ is $\sqrt{\frac{r_{\text {Sire }}^{2}+r_{\text {Dam }}^{2}}{4}}$. A realistic accuracy for a dam EBV $\left(r_{\text {Dam }}\right)$ without considering its own YD in the AMMA model is 0.466 (Table 5). By accounting for YD, this can be considerably increased to 0.704. Assuming an accuracy for the EBV of sires $\left(r_{\text {Sire }}\right)$ of $0.910, r_{Y B}$ equals 0.575 with and 0.511 without the YD of the dam, respectively. This theoretical result is in line with the observed results in our study and indicates that YD should be included in MA-BLUP models.

According to our results, MA-BLUP is a useful tool for the selection among half-sibs in segregating families, even with small QTL effects. For example, the accuracies of young bulls for a QVR of 0.10 were 0.595 in the AM, 0.536 in the MA-BLUP DYD model, and 0.596 in the MA-BLUP DYD-YD model. If only the accuracies are considered, a substantial decrease is observed when moving from the AM to the MA-BLUP DYD model and only a small increase when comparing AM and the MA-BLUP DYD-YD model. However, an explicit analysis of segregating families shows a better differentiation between half-sibs, because the Mendelian sampling variance within families can be partially exploited, and correlations between EBV of relatives decrease (Meuwissen and van Arendonk, 1992). This is especially true for MA-BLUP DYD-YD models. The key requirement to achieve these gains is the ability to produce enough full or half-sib progeny to replace those precluded from progeny testing due to carrying unfavorable QTL alleles (Mackinnon and Georges, 1998).

This study considered only a single trait, whereas breeding goals in dairy cattle consist of a variety of traits. Although QTL have been mapped for many of these traits, in some cases explaining approximately $50 \%$ of the genetic variation of a particular trait (Grisart et al., 2002), the fraction of the variation of the overall breeding goal explained by QTL is likely to be moderate (Schrooten et al., 2005). Nevertheless, preliminary simulation studies for MAS (Schrooten et al., 
2005) show encouraging results. Even when the QTL explained only $5 \%$ of the genetic variance, MAS had a considerable effect on the genetic response.

\section{CONCLUSIONS}

For the estimation of QTL variance components in MA-BLUP models, the use of DYD models is the most appropriate strategy. An appropriate weighting is essential, and the best results were obtained by using DE or EDC as weighting factors. The estimated variance components from DYD-YD models were more biased.

The MA-BLUP evaluations that do not make use of phenotypic data for bull dams will only give benefits in young bull selection when the QTL explain more than $30 \%$ of the additive genetic variance. Even in DYD-YD models, data are still incomplete compared with a conventional AM evaluation. To outweigh the loss of information caused by the 2-step approach, a QVR of approximately $10 \%$ is required. With respect to genetic progress, MA-BLUP should be applied even if QTL effects are small, as long as the costs are justified, because MA-BLUP can always improve the selection within segregating families. As a consequence of the results of this study, MA-BLUP models used to estimate EBV for MAS should include DYD and YD to ensure that MAS improves selection even for moderate QTL effects. Accounting for the different genetic and residual variances in DYD and YD by the additional weight $\mathrm{Y}$ further improved the results for the estimation of variance components and EBV in DYD-YD MABLUP models.

\section{ACKNOWLEDGMENTS}

We gratefully acknowledge financial support from the German Federal Ministry of Education and Research (project FUGATO MAS.-Net, grant no. 0313390F) and of the Förderverein Biotechnologieforschung, Bonn, Germany.

\section{REFERENCES}

Abdel-Azim, G., and A. E. Freeman. 2001. A rapid method for computing the inverse of the gametic covariance matrix between relatives for a marked quantitative trait locus. Genet. Sel. Evol. 33:153-173.

Bennewitz, J., N. Reinsch, S. Paul, C. Looft, B. Kaupe, C. Weimann, G. Erhardt, G. Thaller, Ch. Kühn, M. Schwerin, H. Thomsen, F. Reinhardt, R. Reents, and E. Kalm. 2004a. The DGAT1 K232A mutation is not solely responsible for the milk production quantitative trait locus on the bovine chromosome 14. J. Dairy Sci. 87:431-442.

Bennewitz, J., N. Reinsch, F. Reinhardt, Z. Liu, and E. Kalm. 2004b. Top down preselection using marker assisted estimates of breeding values in dairy cattle. J. Anim. Breed. Genet. 121:307318.

Boichard, D., S. Fritz, M. N. Rossignol, M. Y. Boscher, A. Malafosse, and J. J. Colleau. 2002. Implementation of marker-assisted selection in French dairy cattle. Communication no. 22-03, page 149 in Proc. 7th World Congr. Genet. Appl. Livest. Prod., Montpellier, France.

Druet, T., S. Fritz, D. Boichard, and J. J. Colleau. 2006. Estimation of genetic parameters for quantitative trait loci for dairy traits in the French Holstein population. J. Dairy Sci. 89:4070-4076.

Druet, T., S. Fritz, J. J. Colleau, M. Gautier, A. Eggen, M. N. Rossignol, M. Y. Boscher, A. Malafosse, and D. Boichard. 2005. Genetic markers in breeding programs. The 26th European Holstein and Red Holstein Conference, Prague 2005. http://www. whff.info/pdf/26ehc_prague/druet.pdf Accessed Oct. 10, 2007.

Fernando, R. L., and M. Grossman. 1989. Marker assisted selection using best linear unbiased prediction. Genet. Sel. Evol. 21:467477.

Fikse, W. F., and G. Banos. 2001. Weighting factors of sire daughter information in international genetic evaluations. J. Dairy Sci. 84:1759-1767.

Gilmour, A. R., R. Thompson, and B. R. Cullis. 1995. Average information REML: An efficient algorithm for variance parameter estimation in linear mixed models. Biometrics 51:1440-1450.

Grisart, B., W. Coppieters, F. Farnir, L. Karim, C. Ford, P. Berzi, N. Cambisano, M. Mni, S. Reid, P. Simon, R. Spelman, M. Georges, and R. Snell. 2002. Positional candidate cloning of a QTL in dairy cattle: Identification of a missense mutation in the bovine DGAT1 gene with major effect on milk yield and composition. Genome Res. 12:222-231.

Interbull. 2007. Description of National Genetic Evaluation System and Trend Validation for Production Traits. http://www-interbull. slu.se/national_ges_info2/framesida-ges.htm Accessed Nov. 22, 2007.

Liu, Z., F. Reinhardt, J. Szyda, H. Thomsen, and R. Reents. 2004. A marker assisted genetic evaluation system for dairy cattle using a random QTL model. Interbull Bull. 32:170-174.

Mackinnon, M. J., and M. A. J. Georges. 1998. Marker-assisted preselection of young dairy sires prior to progeny-testing. Livest. Prod. Sci. 54:229-250.

Meuwissen, T. H. E., and J. A. M. van Arendonk. 1992. Potential improvements in rate of genetic gain from marker-assisted selection in dairy cattle breeding schemes. J. Dairy Sci. 75:16511659 .

Rijsdijk, F. V., and P. C. Sham. 2002. Improved estimation of multipoint IBD sharing and multipoint information content by linear regression. Behav. Genet. 32:211-220.

Ruane, J., and J. J. Colleau. 1996. Marker-assisted selection for a sex-limited character in a nucleus breeding population. J. Dairy Sci. 79:1666-1678.

Schrooten, C., H. Bovenhuis, J. A. M. van Arendonk, and P. Bijma. 2005. Genetic progress in multistage dairy cattle breeding schemes using genetic markers. J. Dairy Sci. 88:1569-1581.

Spelman, R. J., and J. A. M. van Arendonk. 1997. Effect of inaccurate parameter estimates on genetic response to marker-assisted selection in an outbred population. J. Dairy Sci. 80:3399-3410.

Szyda, J., Z. Liu, F. Reinhardt, and R. Reents. 2005. Estimation of quantitative trait loci parameters for milk production traits in German Holstein dairy cattle population. J. Dairy Sci. 88:356367.

Thaller, G., W. Krämer, A. Winter, B. Kaupe, G. Erhardt, and R. Fries. 2003. Effects of DGAT1 variants on milk production traits in German cattle breeds. J. Anim. Sci. 81:1911-1918.

VanRaden, P. M., and G. R. Wiggans. 1991. Derivation, calculation, and use of national animal model information. J. Dairy Sci. 74:2737-2746.

Vuori, K., I. Strandén, M. Lidauer, and E. Mäntysaari. 2006. MIX99 - Effective solver for large and complex linear mixed models. Communication no. 27-33, page 254 in Proc. 8th World Congr. Genet. Appl. Livest. Prod., Belo Horizonte, Brazil.

Winter, A., W. Krämer, F. A. O. Werner, S. Kollers, S. Kata, G. Durstewitz, J. Buitkamp, J. E. Womack, G. Thaller, and R. Fries. 2002. Association of a lysine-232/alanine polymorphism in a bovine gene encoding acyl-CoA:diacylglycerol acyltransferase (DGAT1) with variation at a quantitative trait locus for milk fat content. Proc. Natl. Acad. Sci. USA 99:9300-9305. 


\section{APPENDIX}

\section{Variances of DYD and YD}

The aim of 2-step approaches is to use all available information of animals in the MA-BLUP pedigree. In actual-world MA-BLUP evaluations, some models use only DYD (Liu et al., 2004), whereas other consider both DYD and YD (Boichard et al., 2002) as phenotypic information. The expected values of the variance of these types of information are not identical. We propose to apply an additional weight to DYD to ensure a correct combination of DYD and YD.

Because YD are only corrected for fixed effects (VanRaden and Wiggans, 1991), they carry full additive genetic variance $\left(\sigma_{A}^{2}\right)$ and full residual variance $\left(\sigma_{E}^{2}\right): \operatorname{var}(Y D)=\sigma_{A}^{2}+\sigma_{E}^{2}$. DYD are defined as a weighted average of corrected YD of all progeny of a sire, where the correction is for all fixed effects and the breeding values of the mates of a sire (VanRaden and Wiggans, 1991). Ignoring inbreeding, assuming that all mating partners of a sire are known, and only considering 1 record per progeny, the DYD of a bull can be calculated as $D Y D=\frac{\sum_{i=1}^{n}\left(Y D_{i}-1 / 2 E B V_{m_{i}}\right)}{n}$, where $Y D_{i}=$ the $\mathrm{YD}$ of progeny $i ; E B V_{m_{i}}=$ the EBV of the dam of the progeny $i$; and $n=$ the number of progeny used for the calculation of the DYD. As a consequence, a DYD calculated under the assumptions made contains the genetic variance of half the BV of the father $\left(\sigma_{U}^{2}\right)$ and a residual variance component $\left(\sigma_{E^{*}}^{2}\right)$ that is a function of the Mendelian sampling variance $\left(\sigma_{A}^{2} / 2\right)$, the residual variance $\left(\sigma_{E}^{2}\right)$, and the number of progeny (n) that were used for the calculation of the DYD:

$$
\operatorname{var}(D Y D)=\sigma_{U}^{2}+\sigma_{E^{*}}^{2}=\sigma_{A}^{2} / 4+\frac{\left(\sigma_{A}^{2} / 2+\sigma_{E}^{2}\right)}{n} .
$$

To put DYD and YD on the same scales, both should contain full $\sigma_{A}^{2}$ and full $\sigma_{E}^{2}$. Multiplication of DYD by the factor 2 leads to full $\sigma_{A}^{2}$ in DYD:

$$
\begin{gathered}
\operatorname{var}(2 \times D Y D)=4 \times \operatorname{var}(D Y D)= \\
4 \times\left(\sigma_{U}^{2}+\sigma_{E^{*}}^{2}\right)=\sigma_{A}^{2}+\frac{\left(2 \times \sigma_{A}^{2}+4 \times \sigma_{E}^{2}\right)}{n} .
\end{gathered}
$$

As can be seen, $4 \times \sigma_{E^{*}}^{2}=\frac{\left(2 \times \sigma_{A}^{2}+4 \times \sigma_{E}^{2}\right)}{n}$ of $2 \times$ DYD is definitely not equal to $\sigma_{E}^{2}$. The averaging effect of denominator $n$ is usually accounted for by applying weighting factors like DE or EDC that describe the amount of phenotypic information of the progeny that was used for their calculation.

Additionally, $\left(2 \times \sigma_{A}^{2}+4 \times \sigma_{E}^{2}\right)$ of twice the DYD and $\sigma_{E}^{2}$ of the YD have to be on the same scale. Therefore, we suggest an additional weighting factor $\gamma$. Equating $\left(2 \times \sigma_{A}^{2}+4 \times \sigma_{E}^{2}\right)$ and $\sigma_{E}^{2}$ and integrating $\gamma$ leads to: $\sigma_{E}^{2}=\gamma \times\left(2 \times \sigma_{A}^{2}+4 \times \sigma_{E}^{2}\right)$. Setting this equation equal to $\gamma$ gives us the additional weight for twice the DYD:

$$
\gamma=\frac{\sigma_{E}^{2}}{\left(2 \times \sigma_{A}^{2}+4 \times \sigma_{E}^{2}\right)} .
$$

Applying the weighting factor EDC, which is in our situation directly proportional to $n(w \approx n)$, and the additional weight $\gamma$ leads to:

$$
\begin{gathered}
\operatorname{var}(2 \times D Y D)=\sigma_{A}^{2}+\gamma \times w \times \frac{\left(2 \times \sigma_{A}^{2}+4 \times \sigma_{E}^{2}\right)}{n} \approx \\
\sigma_{A}^{2}+\gamma \times\left(2 \times \sigma_{A}^{2}+4 \times \sigma_{E}^{2}\right)=\sigma_{A}^{2}+\sigma_{E}^{2} .
\end{gathered}
$$

The calculation of $\gamma$ requires only the knowledge of variance components. These can easily be estimated from an animal model without QTL effects. 